\title{
Switch of dimensionality of exciton diffusion in aggregates
}

\author{
Steffen Wolter ${ }^{1, *}$, Frank Würthner ${ }^{2}$, Oliver Kühn ${ }^{1}$, and Stefan Lochbrunner ${ }^{1}$ \\ ${ }^{1}$ Institut für Physik, Universität Rostock, Albert-Einstein-Straße 23, 18059 Rostock, Germany \\ ${ }^{2}$ Institut für Organische Chemie and Center for Nanosystems Chemistry, Universität Würzburg, Am \\ Hubland, 97074 Würzburg, Germany
}

\begin{abstract}
The ultrafast exciton dynamics in J-aggregates of a perylene bisimide dye is investigated for temperatures down to $77 \mathrm{~K}$ revealing at low temperatures a decrease of the exciton mobility and a change in the dimensionality.
\end{abstract}

\section{Introduction}

Molecular aggregates attract increasing scientific interest due to their potential for electronic and photonic applications [1]. Especially for the latter the exciton mobility is a crucial factor. J-aggregates can exhibit particularly delocalized and very mobile excitons. Here we study the perylene bisimide dye $N, N$-di[ $N$-(2-aminoethyl)-3,4,5tris(dodecyloxy)benzamide]-1,6,7,12-tetra(4-tert-butylphenoxy)perylene-3,4:9,10-tetracarboxylic acid bisimide (PBI-1, see inset of Fig. 1) which forms stabile J-aggregates in methylcyclohexane $(\mathrm{MCH})$ already at moderate concentrations [1,2]. The photoinduced dynamics is investigated at different temperatures to understand the impact of disorder and thermal activation on the exciton mobility.

\section{Experimental Results and Discussion}

Fused silica cells with a layer thickness of $1 \mathrm{~mm}$ are filled with a $0.09 \mathrm{mM}$ solution of PBI$\mathbf{1}$ in $\mathrm{MCH}$ and placed into a continuous flow cryostat operated with liquid nitrogen. Stationary spectroscopy shows that at this concentration J-aggregates are formed. Pumpprobe absorption measurements are carried out applying for excitation $30 \mathrm{fs}$ long pulses centred at $530 \mathrm{~nm}$ from a non-collinearly phase-matched optical parametric amplifier (NOPA) and as probe a white light continuum generated in a calcium fluoride substrate [3].

Transient absorption spectra of PBI-1 in MCH are depicted in Fig. 1 for three different temperatures. They show a strong negative band due to ground state bleach and stimulated emission. Upon cooling, narrowing of the bands and a solvent induced red shift occur, which is also observed in the stationary spectra. In addition, a positive signal contribution, which is particularly visible at $77 \mathrm{~K}$, exists at the blue side of the dominant band. It is attributed to the excited state absorption from the one-exciton to the two-exciton state.

\footnotetext{
* Corresponding author: steffen.wolter@uni-rostock.de
} 


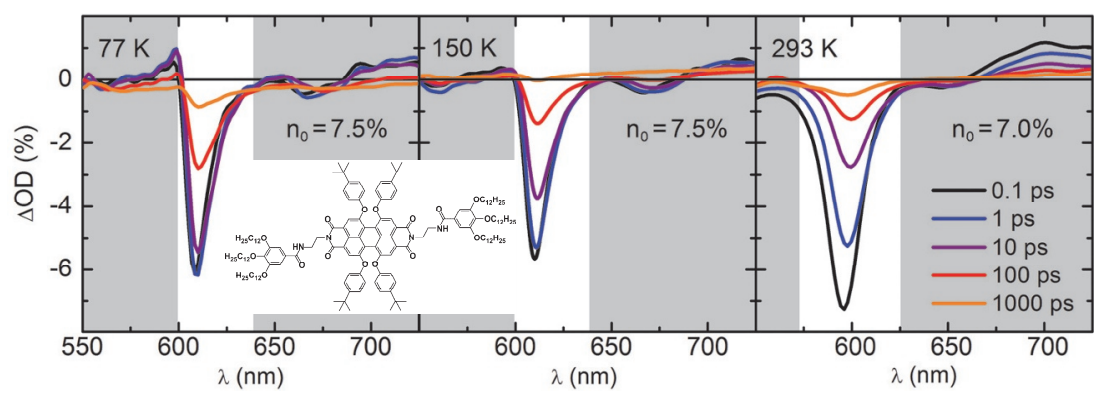

Fig. 1. Transient absorption spectra of PBI-1 aggregates after optical excitation at $530 \mathrm{~nm}$ for different sample temperatures. $n_{0}$ denotes the optically generated exciton density per molecule.

The decay of the signal is non-exponential and accelerates with increasing excitation density. It is dominantly caused by bimolecular exciton-exciton annihilation [2]. Accordingly, the evolution of the time dependent exciton density $n(t)$ is described by the rate equation Equ. 1.

$$
\frac{\mathrm{d} n(t)}{\mathrm{d} t}=-\frac{n(t)}{\tau}-\frac{1}{2} \gamma(t) n^{2}(t)
$$

$\tau$ is the intrinsic lifetime of the excitons and $\gamma(t)$ the bimolecular annihilation rate. The latter depends on the dimensionality and reads for one- and three-dimensional diffusive exciton motion $[2,3]$ :

$$
\gamma_{1 \mathrm{D}}(t)=\frac{1}{a N_{0}} \sqrt{\frac{8 D_{1 \mathrm{D}}}{\pi t}} \quad \gamma_{3 \mathrm{D}}(t)=8 \pi D_{3 \mathrm{D}} \bar{a}\left(1+\frac{\bar{a}}{\sqrt{2 \pi D_{3 \mathrm{D}} t}}\right)
$$

with the molecular density $N_{0}$, the lattice constant $a$ and the diffusion constant $D_{1 \mathrm{D}}$. In the case of three-dimensional motion, $D_{3 \mathrm{D}}$ is the isotropically averaged diffusion constant and $\bar{a}$ the isotropic annihilation interaction distance that should be the lattice constant for next neighbour interaction. Figure 2 shows for the three considered temperatures the time dependent exciton densities as they are obtained from the spectrally integrated transient absorption (white regions in Fig. 1). At room temperature (293 K, green dots) the decay is faster than at lower temperatures and can be described by one-dimensional diffusion driven annihilation (Fig. 2a). The fit of Equ. 1 using the one-dimensional annihilation rate $\gamma_{1 D}$ reproduces the decay dynamics at room temperature very well and yields a diffusion constant of $D_{1 \mathrm{D}}=0.36 \mathrm{~nm}^{2} / \mathrm{ps}$. However, the one-dimensional model does not work for the lower temperatures, see Fig. $2 \mathrm{a}$. Therefore, the three-dimensional model, i.e. $\gamma_{3 \mathrm{D}}$ is applied to these measurements. It works much better for both, the $150 \mathrm{~K}$ and the $77 \mathrm{~K}$ data, but fails at $293 \mathrm{~K}$ (see Fig. 2b). A diffusion constant $D_{3 \mathrm{D}}$ of $0.016 \mathrm{~nm}^{2} / \mathrm{ps}$ is found for $150 \mathrm{~K}$ and of $0.005 \mathrm{~nm}^{2} / \mathrm{ps}$ for $77 \mathrm{~K}$. This reduction of the exciton mobility can be traced back to the decrease of the spectral overlap between absorption and fluorescence.

The stationary absorption spectra show upon cooling no new features pointing to a compact three-dimensional packing of the PBI-1 molecules and the structure responsible for the shape of the spectra is still the one-dimensional aggregate chain [3]. Furthermore, it seems highly unlikely that extended three-dimensional structures are formed for the following reasons. At cold temperatures clear samples are obtained and no pronounced scattering is observed, which would be typical for particles or microcrystals. Additionally, the cooling process is quite fast and the time window, during which the sample is liquid, is 
only a few minutes. During such short times and for structures like those investigated here it is unlikely that extended three-dimensional crystals appear. Rather we expect only small agglomerates, most likely consisting of several intertwined chains. However, making use of Monte-Carlo simulations we find that intertwined nanofibrous structures consisting of a very limited number of PBI-1 aggregate chains can already result in a virtual threedimensional behaviour of the annihilation dynamics [3].



Fig. 2. Time dependent exciton densities as obtained from integrated transient absorption signals together with fits assuming (a) one-dimensional and (b) three-dimensional exciton diffusion.

\section{Conclusions}

The decay of the exciton density in PBI-1 aggregates can be modelled and understood in terms of diffusion driven exciton-exciton annihilation. It is found that the exciton motion is substantially slowed down at low temperatures. The temperature dependence of the fitted exciton diffusion constant resembles thereby almost perfectly the changes in the spectral overlap. This points to an incoherent hopping scenario based on Förster energy transfer. Static disorder in the aggregate chains results in energy barriers, which limit the delocalization of the excitons as well as their incoherent hopping rate and mobility.

Surprisingly, we observe a change in the exciton decay dynamics that could be reproduced by switching from a one-dimensional model used at room temperature to a three-dimensional model for measurements at $150 \mathrm{~K}$ and $77 \mathrm{~K}$. This variation of the exciton decay behaviour is most likely caused by a structural change from one-dimensional chain like aggregates at room temperature to more extended structures at low temperatures. The latter seem to consist of a limited set of weakly interlinked chains as it is frequently observed for dye aggregates often resulting in the formation of organogels.

Financial support by the German Science Foundation via the SFB 652 is gratefully acknowledged.

\section{References}

1. F. Würthner, T. E. Kaiser, and C. R. Saha-Möller, Angew. Chem., Int. Ed. 50, 3376 (2011)

2. S. Wolter, J. Aizezers, F. Fennel, M. Seidel, F. Würthner, O. Kühn, and S. Lochbrunner, New J. Phys. 14 , 105027 (2012)

3. S. Wolter, K. M. Westphal, M. Hempel, F. Würthner, O. Kühn, and S. Lochbrunner, J. Phys. B: At. Mol. Opt. Phys. 50, 184005 (2017) 